\title{
Design of Broadband Transmitarray Unit Cells with Comparative Study of Different Numbers of Layers
}

\author{
Hoda Nematollahi, Jean-Jacques Laurin
}

\author{
J.E. Page, José A. Encinar.
}

\begin{abstract}
Transmitarray antennas are being developed to significantly reduce the mass and the cost of beamforming antennas for satellite communication. A generalized methodology to design low profile transmitarray antennas made of several stacked layers with non-resonant printed phasing elements is presented. A study of the unit cell bandwidth, phase-shift range and tolerances has been conducted considering different numbers of layers. A structure with 3 metallized layers with capacitive and inductive elements enabling a phase range of nearly 360 degrees and low insertion loss is introduced. An implementation on a flexible substrate of a transmitarray with progressive phase shift operating at $19 \mathrm{GHz}$ is used for validation.
\end{abstract}

Index Terms- Multi-layers, spatial phase shifters, transmitarray antenna.

\section{INTRODUCTION}

$\mathrm{T}$ ransmitarrays (TA) can be used in satellite-based telecommunication systems as low profile conformal designs with high radiation efficiency and no feed blockage. They combine the advantages of both reflectors and array antennas and are less sensitive to thermo-elastic distortions than reflectarrays [1].

Different concepts have been used to design TAs, also called discrete lenses. TAs are classified into active (when external control signal is used for the inner circuit configuration) [2,3] and passive regarding the circuitry applied to achieve the phase shift. Typical passive TAs consist of receive and transmit antennas (typically microstrip patch antennas) on opposed layers, connected together using a phase shifting mechanism. The required phase shift can be achieved by transmission lines with variable lengths connecting the elements of receiving and transmitting arrays, or apertures used to couple the arrays of receiving and transmitting microstrip patch antennas together $[4,5]$.

A cascade of phase shifting layers is also used where the phase shift distribution required to synthesize a radiation pattern is achieved by the phase response of the cells transfer function. In most techniques, resonant elements are used with periodicities in the order of half a wavelength $[6,7]$. This large periodicity leads to an increase in sensitivity to angle of incidence and grating lobes. Recently, some authors have presented a class of low-profile frequency selective surfaces that are composed of non-resonant periodic structures [8]. These structures are referred to as miniaturized element frequency selective surface (MEFSSs). This class of selective surfaces is more stable as a function of the incidence angle of the electromagnetic wave. Some studies have been conducted recently on the number of layers in the design of phase shifting surface lens antennas $[9,10]$.

The main objectives in the design of multilayer phase shifters being the increase of the phase range, the minimization of losses and higher stability, a study is conducted in this paper on the sensitivity of TA unit cells made of multiple layers to frequency and spacing variations, using a very simple and effective analysis method regardless of the elements shape. In the following, we will first present the principles of design of a stacked layer transmitarray. In Section III, the proposed phase shifting elements are introduced. A prototype of a 3-layer beam deviating TA is fabricated and measured in Section IV. A comparative study on the stability and phase shifting ranges considering from 2 to 4 layers is then presented in Section V. Finally, conclusions are drawn in Section VI.

\section{PRINCIPLES OF OPERATION AND DESIGN PROCEDURE}

Fig. 1 shows the three-dimensional view of a multiple layer TA. The structure is composed of thin substrate layers with printed periodic capacitive and inductive sub-wavelength elements separated either by air or dielectric material.

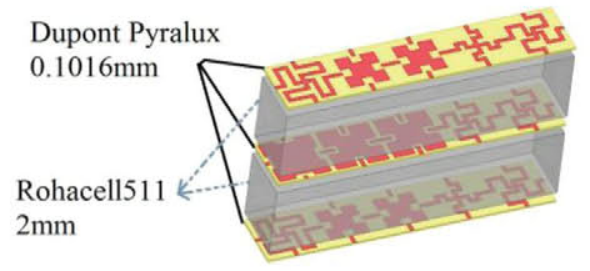

Fig. 1. Drawing of transmitarray panel, with the details of the different types of phasing elements.

Each cell can be represented by the simple equivalent circuit shown in Fig.2 [11], in which each metallized layer is represented by a shunt admittance on a transmission line. For a symmetrical three-layer design, cell layers 1 and 3 have equal 
admittances. The admittance of each element (including the substrate) is obtained by full- wave simulation carried out by the finite element method in Ansys High Frequency Structure Simulator (HFSS). The separations between the layers are represented by the lines sections of length $\mathrm{d}$. A correction due to the electrical length of the substrate thickness is also included in the calculations.

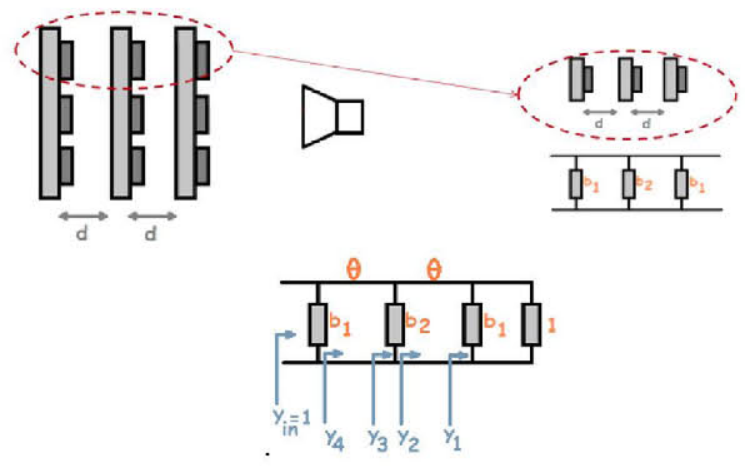

Fig. 2. A simple equivalent circuit model for a 3-layer TA.

The right choice of admittances of the layers and the spacing between them should lead to a database of cells with a transmission phase range of 360 degrees and minimum transmission loss. The matching condition for the input admittance is calculated as a function of values of susceptances b1 and b2 for a fixed separation $d$ and design curves of the transmission phase are drawn as a function of the admittance of the layers for a matched condition. The same procedure is applied for the case of 2 and 4 layers. The transmission phase is selected on the curves and the related values of susceptance for each layer in a matched condition are obtained, determined by the number of layers and the separation between them, regardless of the element shape. Fig. 3 shows design curves for the 3-layer unit cell case, when the separation between the layers is $\lambda / 8$.
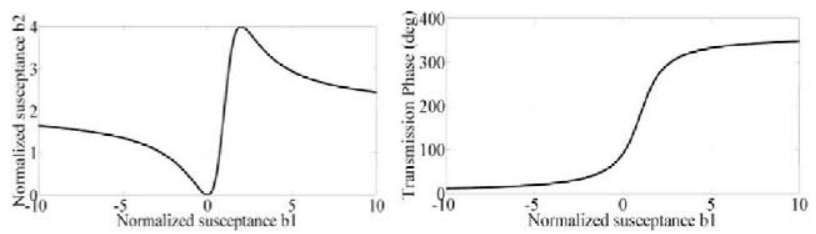

Fig. 3. Transmission phase and matching conditions for 3-layer cells with a separation $d$ of $\lambda / 8$.

It can be seen in the graphs that the shunt elements of the equivalent circuit have to provide inductive and capacitive susceptances. In this example, the 360-degree phase range is nearly achieved, but large values of susceptance are needed. Adequate cells are designed for this function in the next section

\section{DESIGN OF THE UNIT CELL ELEMENT}

The validity of the design curves has been verified through full wave simulations of several representative 3-layer cells. Fig. 4 and Table I show six different types of unit cells which cover the whole inductive and capacitive range. It is observed from the geometry of the cell that they maintain a $90^{\circ}$ rotational symmetry which insures insensitivity to polarization for normal incidence. The desired susceptance of each element is obtained by changing the element's dimensions. As the transmission phase is always calculated in a matched condition for the layers and independent of the geometrical shapes of the elements, the type and size of elements could be chosen inside the range of fabrication tolerances. Regarding the special application of the TA in space applications, interconnecting elements can be privileged to minimize static charge accumulation.

TABLE I

INDUCTIVE AND CAPACITIVE GEOMETRIES

\begin{tabular}{lll}
\hline \hline \multicolumn{1}{c}{ Cell name } & Geometry & Normalized susceptance \\
\hline Inductive cross & $-17 \rightarrow-1.68$ \\
EBG & $-6 \rightarrow-1.9$ \\
Meander & $-1.86 \rightarrow 0.579$ \\
Gap-EBG & $0.56 \rightarrow 4.29$ \\
Patch & $4 \rightarrow 12 \rightarrow \ldots$ \\
Highly capacitive & & \\
Overlapping patches & &
\end{tabular}

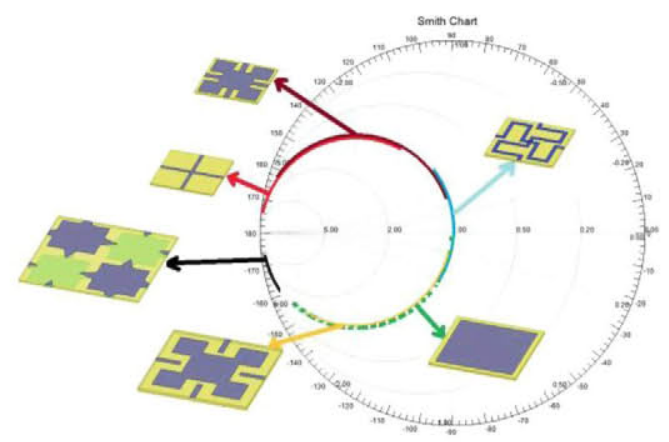

Fig. 4. Inductive and capacitive elements.

Ten different phase shifting cells were designed from the curves of Fig.3, using some of the six susceptance prototypes of Fig. 4. The achievable transmission phase range for a 3layer cell is $321^{\circ}$. Table II shows the exact parameters of each cell. The overall cells consisting of the stacked layers were simulated using full-wave EM simulation HFSS. No optimization was performed and the simulation results prooves the accuracy of the design procedure developed in this paper. As it can be seen in Fig. 5, the insertion loss on the 3-layer cells is minimal for normal incidence at $19 \mathrm{GHz}$. However, the transmission magnitude of some of the cells drops quickly as the frequency is varied around $19 \mathrm{GHz}$ (Fig.6).

The effect of oblique incidence up to $25^{\circ}$ corresponding to the maximum angle of incidence for a focal length-to-diameter ration of 0.9 is simulated and the results for 3-layer cells are shown in Fig. 18. Improvement of the frequency bandwidth of the elements and spacing change tolerance are studied further in section $\mathrm{V}$, as well as the effects of added thickness in errors induced by oblique incidence. 


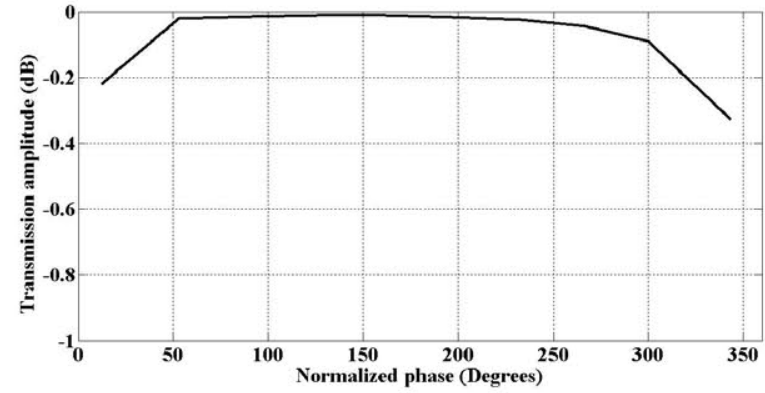

Fig. 5. Amplitude of the transmission coefficient versus normalized transmission phase for different cells obtained from full-wave simulation at $19 \mathrm{GHz}$.

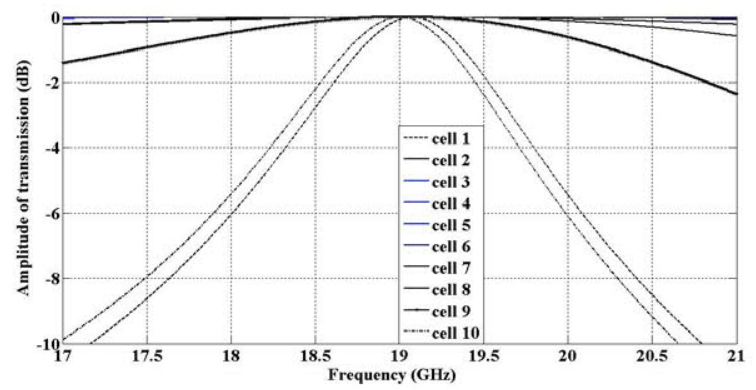

Fig. 6. Transmission magnitude versus frequency for 10 different cells going from a transmission phase of $12^{\circ}$ to $343^{\circ}$, as shown in Table II.

\section{MEASUREMENTS}

The procedure presented in Section II was followed to design a 3-layer TA prototype operating at a center frequency of $19 \mathrm{GHz}$. The TA is implemented on flexible dielectric substrates (Dupont Pyralux) of thickness $0.1016 \mathrm{~mm}$ has $\varepsilon_{r}=3.4$ and $\tan \delta=0.002$. Foam sheets (Rohacell51) are used for the spacers of $2 \mathrm{~mm}(\sim \lambda / 8)$ between the layers. The $40 \times 48$ element TA has a total thickness of $4.3 \mathrm{~mm}$. The unit cell dimensions are slightly smaller than $0.2 \lambda$ at the design frequency. The small size of the cell decreases the quantization error resulting from discretization of the ideal phase profile to be applied on a transmitarray. A linear progressive phase shift is applied on one axis of the TA. The total phase shift on the length of the $7.98 \lambda \mathrm{TA}$ is of $321^{\circ}$, which should cause a beam deviation of $6.42^{\circ}$ for a planar incident wave at normal incidence on the TA. Table II shows the parameters of design of the cells for the ten steps of the progression of phase, as well as the simulated reflection and transmission coefficients of each cell. b1 represents the normalized suscpantance of a cell on the first and third layer (which are identical) and $\mathrm{b} 2$ the normalized susceptance of the elements on the middle layer. A photograph of the fabricated layers is given in Fig.7. Each row is consisted of the element repeated 4 times to have a large enough surface for measurements.

In a first step, a probe performs sweeps on different rows of the TA to measure the transmission phases of different elements and the difference between their delays (Fig.8). Fig. 9 show the measured transmission phases for the ten rows, showing transmission phases from $-25^{\circ}$ to $225^{\circ}$. The phase range of $250^{\circ}$ is smaller than the expected $321^{\circ}$, which is to some extend due to the inaccuracy of the measurement with the waveguide probe but mainly due to the high sensitivity of the elements at the edges which will be explained in section V. However in the middle rows, the difference of the phase shifts is around $36^{\circ}$ which is the expected step of phase difference from the design.

TABLE II

ELEMENTS CHARACTERISTICS OF TA PROTOTYPE

\begin{tabular}{|c|c|c|c|c|c|}
\hline & $B 1$ & $B 2$ & $\begin{array}{c}\text { Phase } \\
\text { shift }\end{array}$ & S11(dB) & S21 (dB, degree) \\
\hline 1 & -6 & 1.289 & 16.5 & -18.4 & $-0.22 \angle 12.4^{\circ}$ \\
\hline 2 & -1.125 & 0.364 & 52.5 & -32.8 & $-0.02 \angle 53.1^{\circ}$ \\
\hline 3 & -0.125 & -0.009 & 88.4 & -48.8 & $-0.015 \angle 91.4^{\circ}$ \\
\hline 4 & 0.375 & 0.307 & 124.4 & -50 & $-0.01 \angle 126^{\circ}$ \\
\hline 5 & 0.725 & 1.1814 & 159.9 & -54.1 & $-0.011 \angle 162^{\circ}$ \\
\hline 6 & 1.025 & 2.246 & 194 & -44.6 & $-0.016 \angle 197^{\circ}$ \\
\hline 7 & 1.375 & 3.206 & 230.6 & -46.8 & $-0.025 \angle 233^{\circ}$ \\
\hline 8 & 1.85 & 3.61 & 266.9 & -30.7 & $-0.044 \angle 266.9^{\circ}$ \\
\hline 9 & 2.7 & 3.34 & 301.8 & -23.2 & $-0.089 \angle 299.8^{\circ}$ \\
\hline 10 & 6 & 2.489 & 337 & -28.6 & $-0.327 \angle 343.5^{\circ}$ \\
\hline & & & & & \\
\hline
\end{tabular}
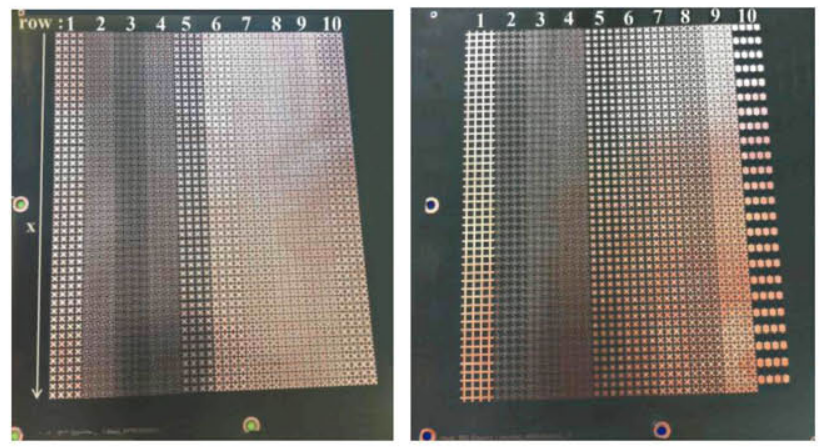

Fig. 7. a) First and third layer, b) Second layer

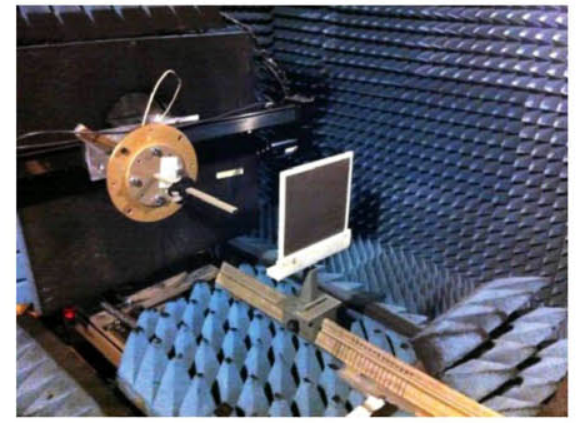

Fig. 8. Measurement setup for rows transmission phase

In order to have a planar incident wave on the transmitarray, a dielectric lens was inserted between the feed horn and the under test TA (Fig. 10). Fig. 11 presents the measured 
radiation pattern of the $\mathrm{TA}$ for $\mathrm{E}$ and $\mathrm{H}$ planes which shows a beam tilt of $6.5^{\circ}$ with $0.89 \mathrm{~dB}$ insertion loss and a crosspolar level of $-35 \mathrm{~dB}$ at the central frequency of $19 \mathrm{GHz}$. A very good agreement is obtained between the measured results and the expected $6.5^{\circ}$ beam deviation. The result demonstrates the viability of the proposed design procedure and element selection.

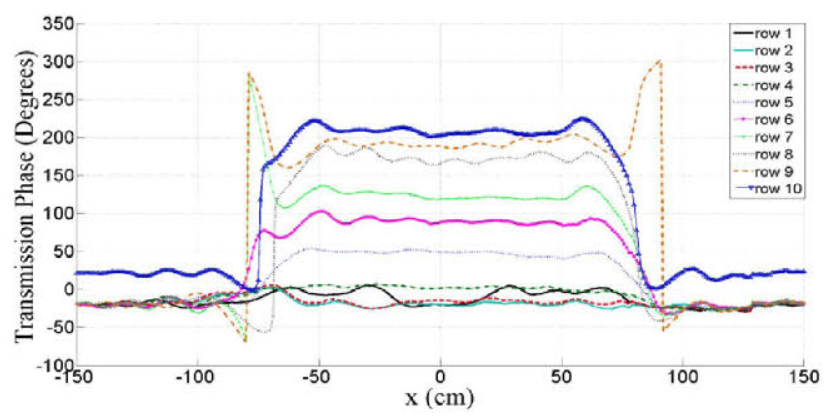

Fig. 9. Measured phases corresponding to a sweep on the rows of Fig. 7

However, the radiation patterns at $18 \mathrm{GHz}$ and $20 \mathrm{GHz}$ have losses of 4 to $5 \mathrm{~dB}$. The bandwidth of the transmitarray is limited by the bandwidth of its elements. It was seen in Fig. 6 that some of the elements have very narrow bandwidth. The next section will address and solve this problem.

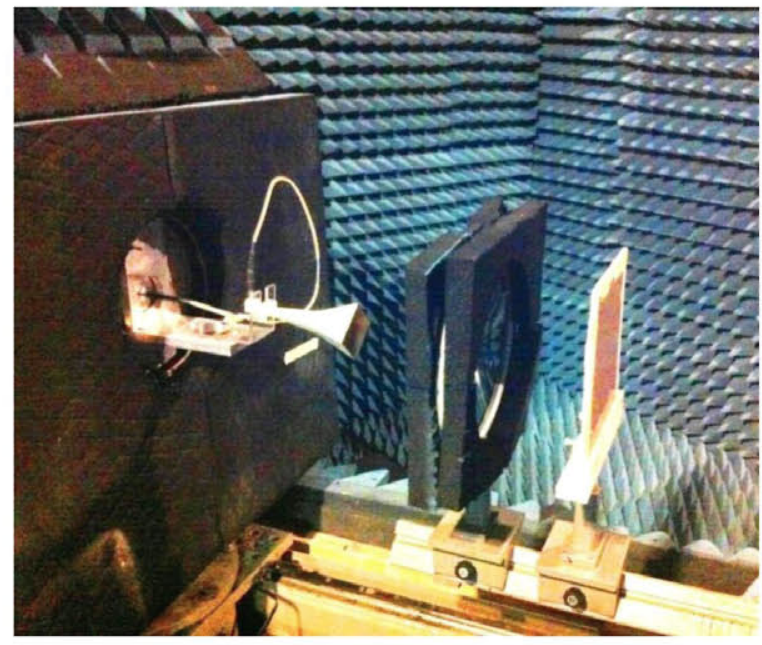

Fig. 10.Far-field measurement setup for the TA in the anechoic chamber

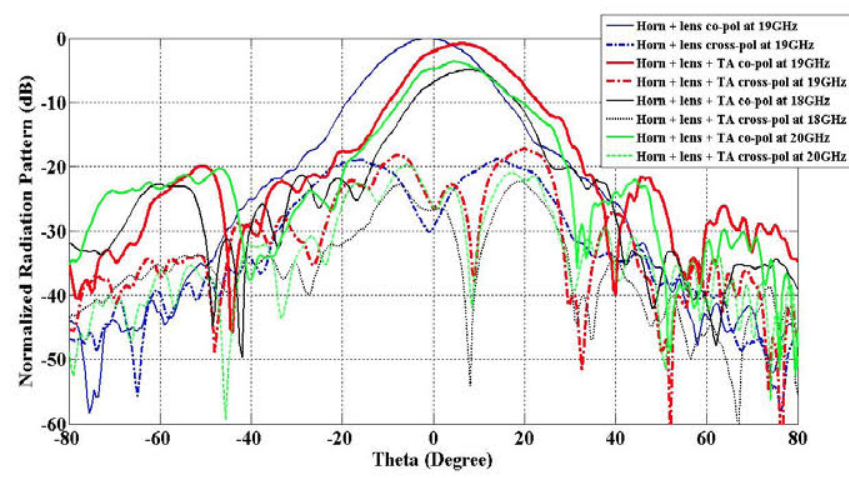

Fig. 11.Prototype measured radiation pattern for $\mathrm{E}$ plane and $\mathrm{H}$ planet from 18 to $20 \mathrm{GHz}$, and compared to the horn and lens only radiation pattern.

\section{SELECTION OF OPTIMAL NUMBER OF LA YERS}

A minimum number of three array layers, including inductive and capacitive elements are necessary to achieve a large enough phase range (close to 321 degrees), while maintaining matched conditions. The phase ranges of 2-layer and 3-layer cells, considered in a periodic array, are compared in Fig. 12.
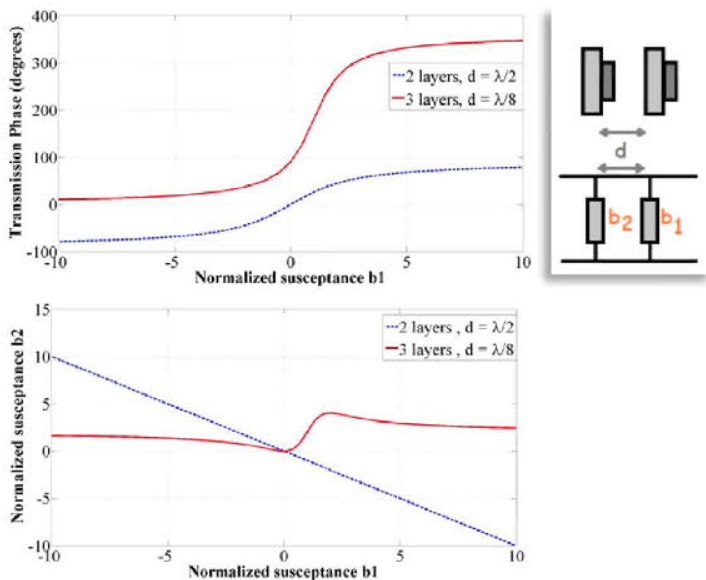

Fig. 12. Transmission phase range and matching condition of 2-layer and 3layer cells.

However, it is shown in Fig. 13 that the matching bandwidth of the cell (less than $3 \mathrm{~dB}$ of insertion loss) and the variation of its transmission phase are dependent on the absolute values of the layers susceptance. High values of susceptance result in a frequency bandwidth as low as $6.3 \%$ for a limit of $3 \mathrm{~dB}$ of transmission loss, whereas keeping the values of $\left|b_{1}\right|$ lower than 3 gives a bandwidth of $22 \%$ to $45 \%$. The transmission phase variation is also reduced to $18^{\circ}$ for $b 1=2$ versus $92^{\circ}$ for $b 2=6$. Limiting the range of susceptances to a maximum normalized absolute value of 3 decreases the phase range to 270 degrees in a 3 -layer structure.
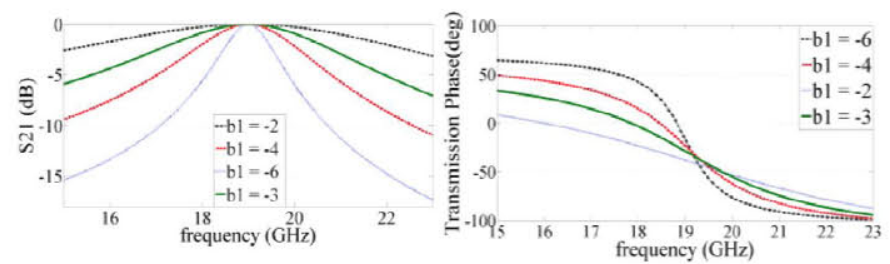

Fig. 13. Transmission loss and phase of unit cell versus frequency for different values of susceptance (b1) for a 3-layer design with $d=\lambda / 8$.

In order to improve the bandwidth of the cells while keeping a large phase range, a 4-layer structure is required. In a 4-layer cell, due to a quadratic equation in the matching condition, two different curves of transmission phase versus susceptance are obtained, which broadens the choices and makes possible the use of cells with small absolute values of susceptance covering a total phase range of 460 degrees (Fig. 14) with stable responses to frequency variations (Fig. 15). 

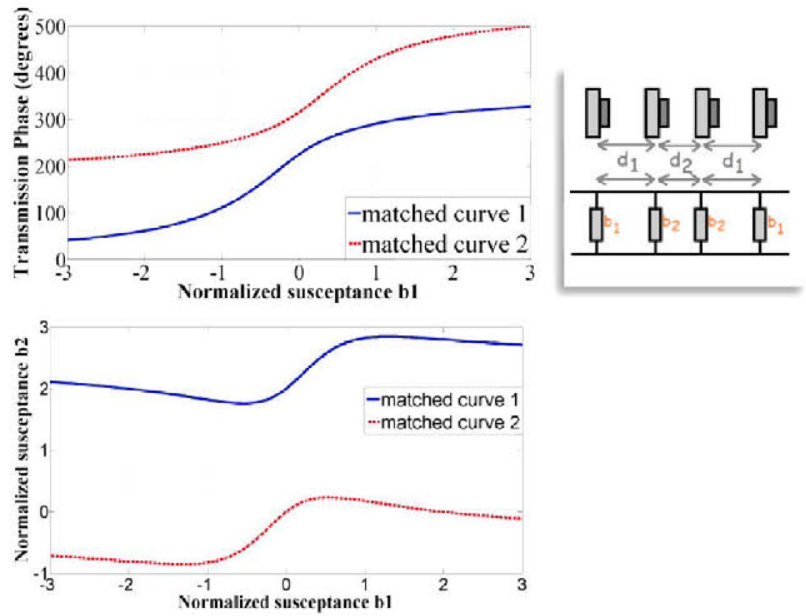

Fig. 14. Transmission phase versus frequency for 4-layer cells with $d_{1}=\lambda / 4, d_{2}=\lambda / 8$

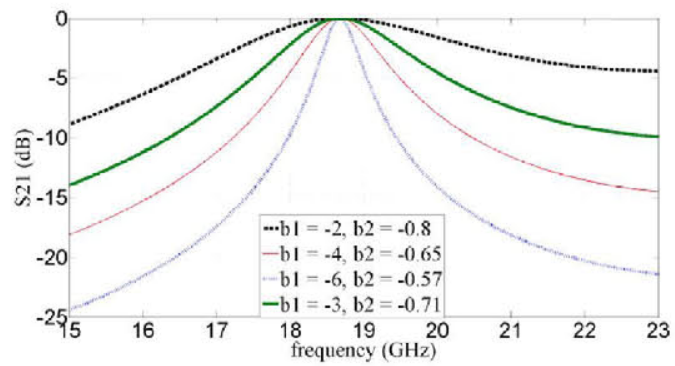

Fig. 15. Transmission loss of unit cell versus frequency for different values of susceptance (b1) for a 4-layer design with $d_{1}=\lambda / 4, d_{2}=\lambda / 8$

Another important consideration in space applications where the panels are subject to extreme changes of temperature and so thermo-elastic distortions of the substrate layers is the effect of such variations on the performance of the TA. A variation of $10 \%$ is applied to the spacing between the layers on 3-layer cells and 4-layer cells. Full-wave simulations give the maximum phase shift error for a change of $\pm 5 \%$ in the spacing in Fig. 16. The 3-layer cells shows varied values of phase errors going up to $110^{\circ}$ which is a very high error of phase introduced in the TA, whereas the 4-layer cells show a better stability and an error around $20^{\circ}$. Figure 17 shows transition losses for a 3-layer cell with $\mathrm{b} 1=-6$ for the lowest phase shift in the range, and the transition loss for the lowest phase shift in a 4-layer cell (bl=-3). In a design where a phase discretization of 3-bits is applied, the highest absolute value of suscpetance is -1.9 which curve shows losses less than $1 \mathrm{~dB}$ in the $10 \%$ variation of spacing.

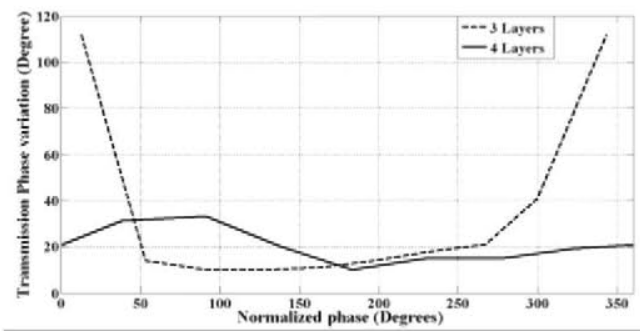

Fig. 16. Transmission phase variations for different phase shifting cell with a change of $\pm 0.1 \mathrm{~mm}$ in the spacing between the layers in a 3-layer cells and 4layer cells.

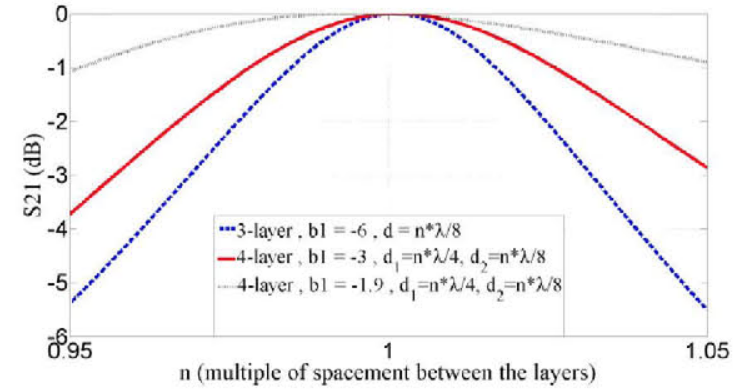

Fig. 17. Transmission loss of unit cell versus change in the spacing between the layers for different values of susceptance (b1) for a 3-layer cell with $d=\lambda / 8$ and 4-layer cells with $d_{1}=\lambda / 4$ and $d_{2}=\lambda / 8$.

The impact of the addition of one layer and the increased thickness of the cells on the sensitivity to the angle of incidence for the 4-layer cell (total thickness of $0.65 \lambda$ ) is studied and compared to a 3-layer cell (total thickness of $0.27 \lambda$ ). As expected from the negative impact of thickness on sensitivity to the angle of incidence, the average loss for elements with low susceptances at an angle of incidence of $25^{\circ}$ is between $0 \mathrm{~dB}$ and $-0.1 \mathrm{~dB}$, whereas for a 4 layer cell, the loss varies between $-0.1 \mathrm{~dB}$ and $-0.6 \mathrm{~dB}$. However, the higher values of susceptance used in the 3-layer cell in order to have a coverage of $321^{\circ}$ of phase shift causes very high losses (up to $-45 \mathrm{~dB}$ ), reducing the phase range of the transmitted signal to $250^{\circ}$. This is not the case for the more stable 4-layer cell. The study of transmission phase errors for oblique incidence shows the same results for the extreme values of susceptance.
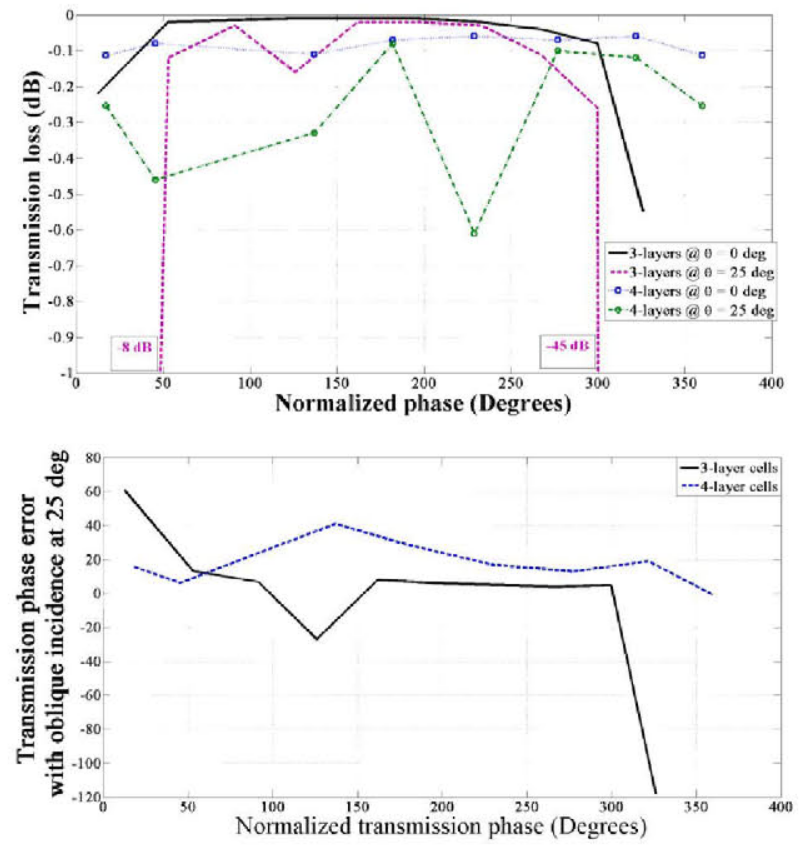

Fig. 18. Simulated results of the effect on transmission loss and transmission phase error of incident waves at oblique incidence of $0^{\circ}$ and $25^{\circ}$ on 3-layer cells with $d=\lambda / 8$ and 4-layer cells with $d_{1}=\lambda / 4$ and $d_{2}=\lambda / 8$.

\section{CONCLUSIONS}

In this work, a very simple and reliable approach has been proposed for the analysis of multiple layer structures as phase shifting cells for transmitarrays. Different types of inductive 
and capacitive elements are presented. Comparison has been conducted for 2, 3 and 4 layers regarding frequency and spacing stability of the cells as well as sensitivity to oblique incidence. A bandwidth of up to $45 \%$ per unit cell is achieved with a maximum loss of $0.4 \mathrm{~dB}$ for the transmitarray elements. The demonstrated limits are generally applicable, independently from the selection of specific element geometry. The variations of the phase range of the cells are validated through full-wave simulations. A beam-deviating transmitarray using 3 layers of sub-wavelength elements was fabricated. Measurement results follows the predictions of the numerical analysis regarding the decreased phase range from $321^{\circ}$ to $250^{\circ}$ because of high losses on high susceptance elements on the limits of the phase range and reinforce the relevance of using 4-layer cells in transmitarray designs.

\section{ACKNOWLEDGMENT}

This has been partially supported by Spanish Ministry of Science and Innovation under projects TEC2010-17567 and CONSOLIDER-INGENIO CSD2008-00068 (TERASENSE). The authors would also like to thank the Fonds de recherche du Québec -- Nature et les technologies (FRQNT) for the international internship scholarship under the CREER Research cluster.

\section{REFERENCES}

[1] M. Barba, E.Carrasco and J.A.Encinar, "Suitable planar transmit-arrays in X-band," First European Conference on. Antennas and Propagation: EuCAP 2006. Nice, France, 6-10 Nov. 2006.

[2] J. Vian, Z. Popovic, Smart lens antenna arrays. IWWW MSD, 2001.
[3] A. Munoz-Acevedo, P. Padilla, M. Sierra-Castaner, "Ku band active transmitarray based on microwave phase shifters," Antennas and Propagation, 2009. EuCAP 2009. 3rd European Conference, pp.1201,1205, 23-27 March 2009

[4] J.Y. Lau, and S.V. Hum, "A Wideband Reconfigurable Transmitarray Element," IEEE Transactions on Antennas and Propagation, vol .60, pp. 1303,1311, March 2012.

[5] H. Kaouach, L. Dussopt, R. Sauleau and T. Koleck, "X-band transmitarrays with linear and circular polarization," Antennas and Propagation: EuCAP 2010, Barcelona, Spain, 12-16 April 2006.

[6] C.G.M. Ryan,.., M.R. Chaharmir, J. Shaker,J.R. Bray, Y.M.M. Antar, and A. Ittipiboon, "A Wideband Transmitarray Using Dual-Resonant Double Square Rings," IEEE Transactions on Antennas and Propagation, vol. 58, pp. 1486-1493, May 2010.

[7] Gagnon, N.; Petosa, A.; McNamara, D.A., "Thin Microwave QuasiTransparent Phase-Shifting Surface (PSS)," Antennas and Propagation, IEEE Transactions on , vol.58, no.4, pp.1193,1201, April 2010

[8] M.A. Al-Joumayly and N. Behdad,, "Wideband Planar Microwave Lenses Using Sub-Wavelength Spatial Phase Shifters," IEEE Transactions on Antennas and Propagation, vol. 59, pp. 4542-4552, Dec. 2011.

[9] N. Gagnon and D. A. McNamara," "Comparison of 2-, 3- and 4-Layer Phase Shifting Surface Lens Antennas,"

[10] A. H. Abdelrahman, A. Z. Elsherbeni and Fan Yang, "Transmission Phase Limit of Multilayer Frequency-Selective Surfaces for Transmitarray Designs," Antennas and Propagation, IEEE Transactions on , vol.62, no.2, pp.690,697, Feb. 2014

[11] M.-A. Joyal, and J.-J. Laurin,"Design of thin circular polarizers," Antennas and Propagation (APSURSI), 2011 IEEE International Symposium on, vol., no., pp.2653, 2656, 3-8 July 2011 